\title{
Reduction of Artifacts in Compressed Videos using Adaptive Fuzzy Filter and Bilateral Filter and Comparison of their Performance
}

\author{
Reeja Mathew \\ Karunya University \\ Coimbatore-641114, India.
}

\begin{abstract}
Compression of videos results in mosquito and flickering artifacts. This paper illustrates the reduction of flickering and mosquito artifacts using adaptive fuzzy filter and bilateral filter and their comparison. Adaptive fuzzy filter is a motion compensated spatiotemporal filter applied to the inter-frame and intra-frame pixels for the reduction of artifacts. Bilateral filter depends on the combination of two parameters - intensity and spatial distance. We have calculated and plotted the graph for PSNR values for the comparison of the two. Experimental results show that the reduction of artifacts using bilateral filter gives better image quality and better robustness. Experimental results illustrating the performance of the two techniques are presented and evaluated.
\end{abstract}

\section{General Terms}

Intensity, spatial, spread parameter, filter, artifact

\section{Keywords}

Artifact reduction, adaptive fuzzy filter, bilateral filter.

\section{INTRODUCTION}

The widely used video compression is normally based on block based DCT technique. DCT exhibits good energy compaction, low computational complexity and de-correlation properties. The main drawback of DCT based compression in video is the formation of blocking, mosquito and flickering artifacts. Blocking artifacts is characterized by visually noticeable changes in pixel values along block boundaries Mosquito artifacts is caused due to the edge busyness in successive still images appear in sequence as a shimmering blur of dots around edges. This artifact come from ringing artifacts when many compressed frames displayed in a sequence. Flicker artifact [1] happen due to the noticeable temporal discontinuity around the intra-frame. It is usually visible more in smooth areas of the image and particularly visible in large groups of pixels. Video quality can be significantly improved by decreasing the mosquito and flickering artifacts. The inconsistency in quality over frames is a temporal distortion caused by quantizing the residual signals [2].

Numerous algorithms have been proposed to alleviate these artifacts in compressed images/videos. For the reduction of artifacts, an effective post-processing method [3] and [4] such as linear low pass filter was used. Most of the de-noising methods are frame based enhancement. Projections onto convex set based method were proposed with multiframe constraint in [5] to reduce artifacts. In [6], flickering artefact term is used to find the optimal prediction and block size mode for intra-frame coding. A similar method was introduced in [7] for flickering reduction in Motion JPEG 2000. In [8] the spatiotemporal median filter for non-motion blocks was used to deal with characteristics of mosquito artifacts and this method cannot achieve better enhancement. A trained bilateral filter was explained in [9] but it requires complicated training based approach and used only for image enhancement. Bilateral filter was illustrated in [10] for the reduction of blocking artifacts in images.

In this paper we are using adaptive fuzzy filter and the bilateral filter to reduce artifacts in compressed videos. The spread parameter of the adaptive fuzzy filter depends on the motion compensated vector and that of the bilateral filter depends on the intensity domain and spatial domain. The performance of both the filter techniques is then evaluated by calculating the PSNR values. This paper is organized as follows. Section II deals with reduction of artifacts using adaptive fuzzy filter. The concept of bilateral filter for artifact removal is introduced in Section III. Section IV presents simulation results of two methods and comparison of their performance. Finally, section $\mathrm{V}$ gives concluding remarks.

\section{REDUCTION OF ARTIFACTS USING ADAPTIVE FUZZY FILTER}

The basic idea behind adaptive fuzzy compensated spatiotemporal filter is to increase the correlation between pixels and making surrounding frames motion compensated before applying the filter. Assume that an adaptive fuzzy filter $h[10]$ is is applied to a set $\Omega$ of neighboring pixels $x^{\prime \prime}\left[t+t^{\prime}, i+i^{\prime}, j+j^{\prime}\right]$ centered by the pixel of interest $x^{\prime}[t, i, j]$. 
$x^{\prime \prime}[t, i, j]=\frac{\sum_{\left[t^{\prime}, i^{\prime}, j^{\prime}\right]} h\left[t^{\prime}, i^{\prime}, j^{\prime}\right] x^{\prime}\left[t+t^{\prime}, i+i^{\prime}, j+j^{\prime}\right]}{\sum_{\left[t^{\prime}, i^{\prime}, j^{\prime}\right] \circledast \Omega} h\left[t^{\prime}, i^{\prime}, j^{\prime}\right]}$

where $h\left[t^{\prime}, i^{\prime}, j^{\prime}\right]$ is the Gaussian membership function and fuzzy filter co-efficient for the neighboring pixels at the location $\left[t^{\prime}, i^{\prime}, j^{\prime}\right]$ from the input $x^{\prime}[t, i, j]$.

$h\left[t^{\prime}, i^{\prime}, j^{\prime}\right]=\exp \left(-\frac{\left(x^{\prime}\left[t+t^{\prime}, i+i^{\prime}, j+j^{\prime}\right]-x^{\prime}[t, i, j]\right)^{2}}{2 \sigma^{2}\left[t^{\prime}, i^{\prime}, j^{\prime}\right]}\right)$

and

$$
\sigma[t, i, j]=F[t, i, j] \times \sigma_{m}[i, j]
$$

is the spread parameter of the filter with amplitude $\sigma_{m}$ and scaling factor $F$.

In the adaptive fuzzy filter, chroma components are upsampled to the same size of luma component. Three frames are given for upsample the chroma components, i.e., $x[t+\mathrm{T}], x[t]$, and $x[t-\mathrm{T}]$. It is enhanced by isotropic fuzzy filter and given to the motion compensated fuzzy filter. The fuzzy filter applied strongest filtering to the pixels in surrounding motion compensated frames and weaker in other positions. The scaling factor is estimated by [10]

$$
F\left[t^{\prime}, i^{\prime}, j^{\prime}\right]=\frac{\sigma_{12}}{\sigma_{1} \sigma_{2}}
$$

where

$$
\begin{gathered}
\sigma_{12}=\sum_{\left[i_{o}, j_{o}\right] \varepsilon V} x^{\prime}\left[t+t^{\prime}, i+i_{o}, j+j^{\prime}+j_{o}\right] \\
\sigma_{1}=\sqrt{\sum_{\left[i_{0}, j_{0}\right] \varepsilon V} x^{\prime}\left[t+t^{\prime}, i+i^{\prime}+i_{0}, i+j^{\prime}+j_{0}\right.} \\
\sigma_{2}=\sqrt{\sum_{\left[i_{0}, j_{0}\right] \varepsilon V} x^{\prime}\left[t, i+i_{0}, j+j_{0}\right]^{2}}
\end{gathered}
$$

$\mathrm{V}$ is the window centered on the pixel of interest. Motion compensated metric is used for evaluating the flickering artifacts. This metric considers the motion of the moving object. The flickering of the $[\mathrm{r}, \mathrm{s}]^{\text {th }}$ block was calculated by sum of square difference (SSD) between original frame I and compressed frame $X$. $S S D_{d i f}[t, r, s]=\frac{\sum_{[i, j] \varepsilon[r, s]^{\text {thblock }}}(D[t, i, j]-D[t-1, i+\Delta i, j+\Delta j])^{2}}{S S D_{\text {org }}[t, r, s]}$

where $[\Delta i, \Delta j]$ is the motion vector of $[\mathrm{r}, \mathrm{s}]^{\text {th }}$ block.
$D[t, i, j]=I[t, i, j]-X[t, i, j]$

If the value of $\mathrm{SSD}_{\text {dif }}$ is less, then less artifacts will be occurred. The metric $\mathrm{S}$ for the whole frame can be computed as

$S(t)=\frac{1}{L_{S S D_{\text {org }}}[t, r, s] \leq \varepsilon} \sum_{d i f}[t, r, s]$

The amplitude $\sigma_{m}$ is calculated using the equation

$\sigma_{m}[i, j]=\sigma_{0}\left((1-\gamma)\left(\frac{S T D\left(x[i, j]-S T D_{\min }\right.}{S T D_{\max }-S T D_{\min }}\right)+\gamma\right)$

where $\sigma_{0}$ is a constant i.e.,15. STD max and STD min are maximum and minimum value of $\operatorname{STD}(x[i, j])$

\section{REDUCTION OF ARTIFACTS USING BILATERAL FILTER}

The bilateral filter is a filter used for artifact reduction. In [9] bilateral filter is used for the removal of artifacts in images. In this bilateral filter is used in compressed videos and the comparison of the performance with adaptive fuzzy filter. Bilateral filtering is done by calculating the weighted averaging of its neighbouring pixels and modifies the current pixel values. The weights can be determined by using two parametersintensity and spatial distance.The output of the bilateral filter is

$S(K)=\frac{1}{F} \sum_{i \varepsilon \tau(k)} e^{-\frac{\|i-k\|^{2}}{2 \sigma_{s}^{2}}} e^{-\frac{\|s(i)-s(k)\|^{2}}{2 \sigma_{i}^{2}}} S(i)$

$\sigma_{i}$ and $\sigma_{s}$ controls weighting average in intensity and spatial domain.

$$
F=\sum_{i \varepsilon \tau(k)} e^{-\frac{\|i-k\|^{2}}{2 \sigma_{s}^{2}}} e^{-\frac{\|s(i)-s(k)\|^{2}}{2 \sigma_{l}^{2}}}
$$

where $\mathrm{F}$ is the normalization factor and $\tau(k)$ is a square of window centred at $S(k)$. In the experiments, $\sigma_{i}$ was found to be linearly related average of the first $3 \times 3$ values from quantization table Q. Empirical formula of intensity domain is

$$
\sigma_{i}=\left(Q_{a v} \times 0.3241\right)+3.1530
$$

The spatial domain $\sigma_{s}$ can found by using $\sigma_{x}$ in the horizontal direction (6) and $\sigma_{y}$ in the vertical direction (7). 
$\sigma_{x}(i, j)=5 \times(\min (a b s(4-i), a b s(i-5))+0.5)$

$\sigma_{y}(i, j)=5 \times(\min (a b s(4-j), a b s(i-4))+0.5)$

The spread parameter $\sigma_{s}(i, j)$ can be obtained by multiplying $\sigma_{x}$ and $\sigma_{y}$.

$$
\sigma_{s}(i, j)=\sigma_{x} \times \sigma_{y}
$$

The resultant matrix is formed for $8 \times 8$ block is as below

$\sigma_{s}(i, j)=\left[\begin{array}{llllllll}240.2 & 162.7 & 85.2 & 7.7 & 7.7 & 85.2 & 162.7 & 240.2 \\ 162.7 & 110.2 & 57.7 & 5.2 & 5.2 & 57.7 & 110.2 & 162.7 \\ 85.25 & 57.75 & 30.2 & 2.7 & 2.7 & 30.2 & 57.75 & 85.25 \\ 7.750 & 5.250 & 2.75 & 0.2 & 0.2 & 2.75 & 5.250 & 7.750 \\ 7.750 & 5.250 & 2.75 & 0.2 & 0.2 & 2.75 & 5.250 & 7.750 \\ 85.25 & 57.75 & 30.2 & 2.7 & 2.7 & 30.2 & 57.75 & 85.25 \\ 162.7 & 110.2 & 57.7 & 5.2 & 5.2 & 57.7 & 110.2 & 162.7 \\ 240.2 & 162.7 & 85.2 & 7.7 & 7.7 & 85.2 & 162.7 & 240.2\end{array}\right]$

\section{EXPERIMENTAL RESULTS}

The algorithm is implemented in MATLAB. Simulations are used to demonstrate the effectiveness of the bilateral filter in compressed videos. Here we are using news as the input video. Simulation of adaptive fuzzy filter is done by finding spread parameter and detects the flickering artifacts using motion compensated vectors. The output of adaptive fuzzy filter is shown in Fig.(1).Smaller the $S S D_{d i f}$, smaller the artifacts, so less filtering is applied. Bilateral filter is applied to the compressed videos. It depends on the variation in intensity and spatial domain. The ouput video using bilateral filter in Fig.(2) achieve significant improvement in visual quality and PSNR. In Fig.(3), a graph for the PSNR value for different frames in two methods is shown to illustrate the performance of the two filters. It summarizes the PSNR value of the resultant video using bilateral filter is high when compared with adaptive fuzzy filter.

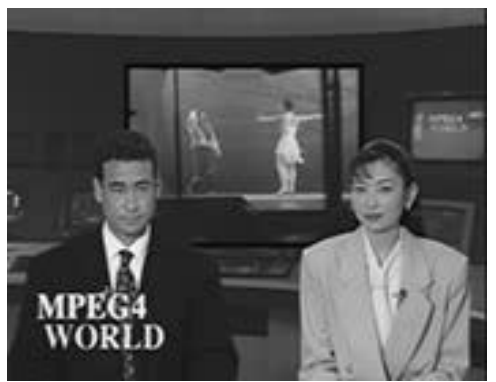

(a)

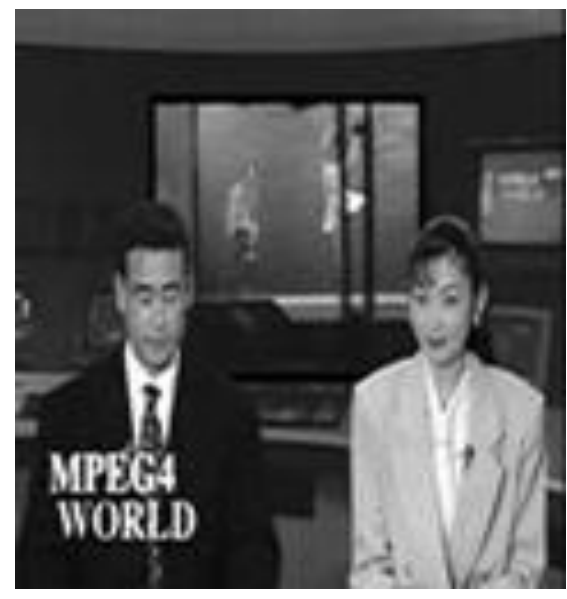

(b)

Fig.1.Adaptive fuzzy filter. (a) Input (b) Output

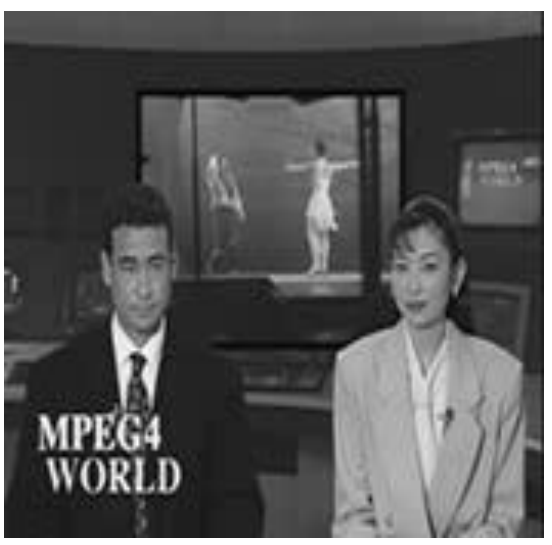

(a)

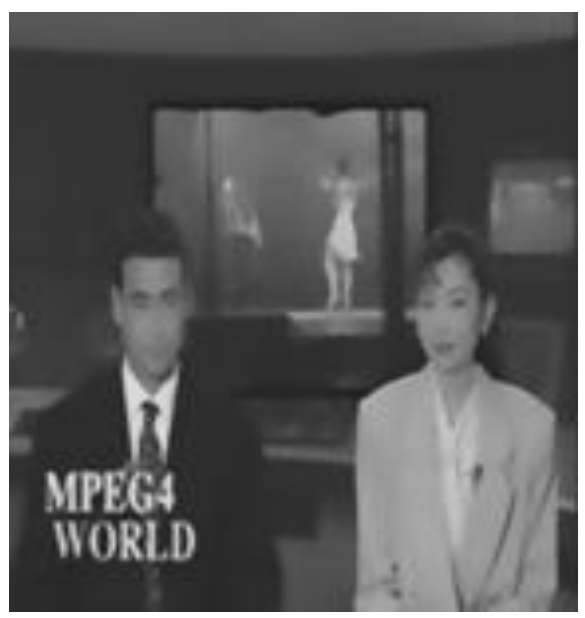

(b)

Fig.2.Bilateral Filter. (a) Input (b) Output 


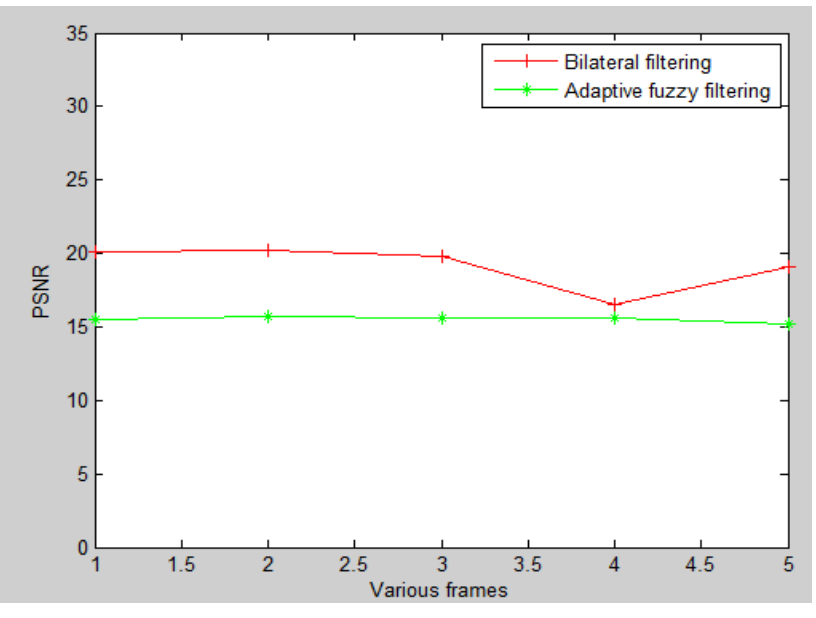

Fig.3.. Graph for different values of psnr for different frames. Red line indicates bilateral filter and green line indicates adaptive fuzzy filter.

\section{CONCLUSION}

Based on the theoretical analysis of blocking and ringing artifacts, increase in the video quality has been analyzed using two methods. Adaptive fuzzy filter has high computational complexity compared to bilateral filter. Bilateral filter is applied for video and analysis its performance based on the intensity domain and spatial domain. The PSNR values for both the techniques has been computed and plotted and we get higher PSNR value in case of bilateral filter which shows that the reduction of flickering and mosquito aritfacts and improved video quality.

\section{REFERENCE}

[1] M. Kaneko, Y. Hatori, and A. Koike, "Improvements of transform coding algorithm for motion-compensated interframe prediction errors-dct/sq coding," IEEE Journal on Selected Areas in Communications, vol. 5, pp. 1068-1078, August 1987.
[2] X. Fan, W. Gao, Y. Lu, and D. Zhao, "Flicking reduction in all intra frame coding," Joint Video Team of ISO/IEC MPEG and ITU-T VCEG, JVT-E070, October 2002.

[3] Deqing Sun and Wai-Kuen Cham,"An effective postprocessing method for low bit rate block dct coded Images," Dept. of EE, the Chinese University of Hong Kong, vol. 1-4244-0728-1, pp. I-705-I708, ICASSP 2007.

[4] Aria Nosratinia, “ Post-Processing of JPEG-2000 Images to Remove Compression Artifacts", IEEEsignal processing letters, 2002.

[5] B.K. Gunturk, Y. Altunbasak, and R. M. Mersereau, "Multiframe blocking-artifact reduction for transformcoded video," IEEE Trans. Circuits Syst. Video Technol., vol. 12, pp. 276-282, April 2002.

[6] S. Sakaida, K. Iguchi, S. Gohshi, and Y. Fujita, "Adaptive quantization control for reducing flicker of AVC/H.264 intra frames," presented at the Picture Coding Symp, Dec. 2004.

[7] A. Leontaris, Y. Tonomura, T. Nakachi, and P. Cosman, "Flicker suppression in JPEG2000 using segmentationbased adjustment of block truncation lengths," in Proc. IEEE Int. Conf. Acoustics, Speech and Signal Processing, Apr. 2007, vol. 1, pp. 1117-1120.

[8] S. DelCorso, C. Miro, and J. Jung, "Mnr: a novel approach to correct mpeg temporal distortions," IEEE Transactions on Consumer Electronics, vol. 49, pp. 229-236, February 2003.

[9] H.Hu and G.de Haan, "Trained bilateral filters and applications to coding artifacts reduction," in Proc .IEEE Int. Conf. Image Processing, vol.1, p.325328, 2007.

[10] J. V. K. Nath and D. Hazarika and A. Mahanta, "Blocking Artifacts Reduction Using Adaptive Bilateral Filtering ”,IEEE Trans. ,vol..1, pp.4244-7138-6.2010

[11] Dũng T. Võ, Student Member, IEEE, Truong Q. Nguyen, Fellow, IEEE, Sehoon Yea, Member, IEEE, and Anthony Vetro, Senior Member, IEEE, "Adaptive Fuzzy Filtering for Artifact Reduction in Compressed Images and Videos" IEEE Transaction on Image Processing ,vol 18, No.6, June 2009 